\title{
Inequalities of Hermite-Hadamard and Bullen Type for $A H$-Convex Functions
}

\author{
Mahir Kadakal $^{1 *}$ and İmdat İşcan ${ }^{1}$ \\ ${ }^{1}$ Department of Mathematics, Faculty of Science and Arts, Giresun University, Giresun, Turkey \\ ${ }^{*}$ Corresponding author
}

\section{Article Info}

Keywords: Convex function, arithmeticharmonically convex function, HermiteHadamard and Bullen type inequalities. 2010 AMS: 26A51, 26D15.

Received: 30 April 2019

Accepted: 3 July 2019

Available online: 30 September 2019

\begin{abstract}
In this paper, by using an integral identity some new general inequalities of the HermiteHadamard and Bullen type for functions whose second derivatives in absolute value at certain power are arithmetically-harmonically convex are obtained. Some applications to special means of real numbers are also given.
\end{abstract}

\section{Introduction}

Definition 1.1. A function $f: I \subseteq \mathbb{R} \rightarrow \mathbb{R}$ is said to be convex if the inequality

$$
f(t x+(1-t) y) \leq t f(x)+(1-t) f(y)
$$

valids for all $x, y \in I$ and $t \in[0,1]$. If this inequality reverses, then $f$ is said to be concave on interval $I \neq \varnothing$. This definition is well known in the literature.

Convexity theory has appeared as a powerful technique to study a wide class of unrelated problems in pure and applied sciences.

Theorem 1.2. Let $f: I \subseteq \mathbb{R} \rightarrow \mathbb{R}$ be a convex function defined on the interval I of real numbers and $a, b \in I$ with $a<b$. The inequality

$$
f\left(\frac{a+b}{2}\right) \leq \frac{1}{b-a} \int_{a}^{b} f(x) d x \leq \frac{f(a)+f(b)}{2}
$$

holds.

The inequality (1.1) is known in the literature as Hermite-Hadamard integral inequality for convex functions. Moreover, it is known that some of the classical inequalities for means can be derived from (1.1) for appropriate particular selections of the function $f$. See [3, 5, 8, 9], for the generalizations, improvements and extensions of the Hermite-Hadamard integral inequality.

Theorem 1.3. Suppose that $f:[a, b] \rightarrow \mathbb{R}$ is a convex function on $[a, b]$. Then, the inequalities are obtained:

$$
\begin{aligned}
f\left(\frac{a+b}{2}\right) & \leq \frac{1}{2}\left[f\left(\frac{3 a+b}{4}\right)+f\left(\frac{a+3 b}{4}\right)\right] \\
& \leq \frac{1}{b-a} \int_{a}^{b} f(x) d x \\
& \leq \frac{1}{2}\left[f\left(\frac{a+b}{2}\right)+\frac{f(a)+f(b)}{2}\right] \leq \frac{f(a)+f(b)}{2} .
\end{aligned}
$$

Email addresses and ORCID numbers: mahirkadakal@gmail.com, https://orcid.org/0000-0002-0240-918X (M. Kadakal), imdati@yahoo.com, https://orcid.org/00000001-6749-0591 (í. İşcan), 
The third inequality in (1.2) is known in the literature as Bullen's inequality.

Definition 1.4 ([2,10]). A function $f: I \subset \mathbb{R} \rightarrow(0, \infty)$ is said to be arithmetic-harmonically $(A H)$ convex function if for all $x, y \in I$ and $t \in[0,1]$ the inequality

$$
f(t x+(1-t) y) \leq \frac{f(x) f(y)}{t f(y)+(1-t) f(x)}
$$

holds. If the inequality (1.2) is reversed then the function $f(x)$ is said to be arithmetic-harmonically (AH) concave function.

Readers can find more informations on arithmetic-harmonically convex functions in $[1,2,4,6,7,10]$ and references therein.

In order to establish some integral inequalities of Hermite-Hadamard type for arithmetic-harmonically convex functions, the following lemma [4] will be used.

Lemma 1.5 ([4]). Let $f: I \subset \mathbb{R} \rightarrow \mathbb{R}$ be a twice differentiable mapping on $I^{\circ}$ such that $f^{\prime \prime} \in L[a, b]$, where $a, b \in I^{\circ}$ with $a<b$, then the following identity holds:

$$
J_{n}(f, a, b)=\sum_{k=1}^{n} \frac{(b-a)^{2}}{2 n^{3}}\left[\int_{0}^{1} t(1-t) f^{\prime \prime}\left(\begin{array}{c}
t\left(\frac{1+n-k}{n} a+\frac{k-1}{n} b\right) \\
+(1-t)\left(\frac{n-k}{n} a+\frac{k}{n} b\right)
\end{array}\right) d t\right]
$$

for all $n \in \mathbb{N}$, where

$$
J_{n}(f, a, b)=\sum_{k=1}^{n} \frac{1}{2 n}\left[f\left(a+\frac{(k-1)(b-a)}{n}\right)+f\left(a+\frac{k(b-a)}{n}\right)\right]-\frac{1}{b-a} \int_{a}^{b} f(x) d x .
$$

In this study, using Hölder integral inequality and the identity (1.4) in order to provide inequality for functions whose first derivatives in absolute value at certain power are arithmetic-harmonically-convex functions.

Throughout this paper, for shortness, the following notations will be used for special means of two nonnegative numbers $a, b$ with $b>a$ :

1. The arithmetic mean

$$
A:=A(a, b)=\frac{a+b}{2}, \quad a, b>0,
$$

2. The geometric mean

$$
G:=G(a, b)=\sqrt{a b}, \quad a, b \geq 0
$$

3. The harmonic mean

$$
H:=H(a, b)=\frac{2 a b}{a+b}, \quad a, b>0,
$$

4. The logarithmic mean

$$
L:=L(a, b)=\left\{\begin{array}{cc}
\frac{b-a}{\ln b-\ln a}, & a \neq b \\
a, & a=b
\end{array} ; a, b>0\right.
$$

5. The $p$-logarithmic mean

$$
L_{p}:=L_{p}(a, b)=\left\{\begin{array}{ccr}
\left(\frac{b^{p+1}-a^{p+1}}{(p+1)(b-a)}\right)^{\frac{1}{p}}, & a \neq b, p \in \mathbb{R} \backslash\{-1,0\} ; a, b>0 . \\
a, & a=b &
\end{array}\right.
$$

These means are often used in numerical approximation and in other areas. However, the following simple relationships are known in the literature:

$$
H \leq G \leq L \leq I \leq A
$$

It is also known that $L_{p}$ is monotonically increasing over $p \in \mathbb{R}$, denoting $L_{0}=I$ and $L_{-1}=L$. In addition,

$$
A_{n, k}=A_{n, k}(a, b)=\frac{1+n-k}{n} a+\frac{k-1}{n} b, n \in \mathbb{N}, k=1,2, \ldots, n,
$$

and $B(\alpha, \beta)$ is the classical Beta function which may be defined by

$$
B(\alpha, \beta)=\int_{0}^{1} t^{\alpha-1}(1-t)^{\beta-1} d t, \alpha, \beta>0 .
$$




\section{Main results}

Theorem 2.1. Let $f: I \subset(0, \infty) \rightarrow(0, \infty)$ be a twice differentiable mapping on $I^{\circ}, n \in \mathbb{N}$ and $a, b \in I^{\circ}$ with $a<b$ such that $f^{\prime \prime} \in L_{1}[a, b]$ and $\left|f^{\prime \prime}\right|$ are an arithmetic-harmonically convex function on the interval $[a, b]$, then the following inequalities hold:

i) If $\left|f^{\prime \prime}\left(A_{n, k+1}\right)\right|-\left|f^{\prime \prime}\left(A_{n, k}\right)\right| \neq 0$, then

$$
\begin{aligned}
& \left|J_{n}(f, a, b)\right| \leq \sum_{k=1}^{n} \frac{(b-a)^{2}}{2 n^{3}} \frac{\left|f^{\prime \prime}\left(A_{n, k}\right)\right|\left|f^{\prime \prime}\left(A_{n, k+1}\right)\right|}{\left(\left|f^{\prime \prime}\left(A_{n, k+1}\right)\right|-\left|f^{\prime \prime}\left(A_{n, k}\right)\right|\right)^{2}} \\
& \times\left[A\left(\left|f^{\prime \prime}\left(A_{n, k}\right)\right|,\left|f^{\prime \prime}\left(A_{n, k+1}\right)\right|\right)-\left|f^{\prime \prime}\left(A_{n, k}\right)\right|\left|f^{\prime \prime}\left(A_{n, k+1}\right)\right| L^{-1}\left(\left|f^{\prime \prime}\left(A_{n, k}\right)\right|,\left|f^{\prime \prime}\left(A_{n, k+1}\right)\right|\right)\right]
\end{aligned}
$$

ii) If $\left|f^{\prime \prime}\left(A_{n, k+1}\right)\right|-\left|f^{\prime \prime}\left(A_{n, k}\right)\right|=0$, then

$$
\left|J_{n}(f, a, b)\right| \leq \sum_{k=1}^{n} \frac{(b-a)^{2}}{12 n^{3}}\left|f^{\prime \prime}\left(A_{n, k+1}\right)\right| .
$$

Proof. i) Let $\left|f^{\prime \prime}\left(A_{n, k+1}\right)\right|-\left|f^{\prime \prime}\left(A_{n, k}\right)\right| \neq 0$. From the Lemma 1.5 and the properties of modulus, the inequality can be written:

$$
\begin{aligned}
\left|J_{n}(f, a, b)\right| & =\left|\sum_{k=1}^{n} \frac{1}{2 n}\left[f\left(a+\frac{(k-1)(b-a)}{n}\right)+f\left(a+\frac{k(b-a)}{n}\right)\right]-\frac{1}{b-a} \int_{a}^{b} f(x) d x\right| \\
& =\left|\sum_{k=1}^{n} \frac{(b-a)^{2}}{2 n^{3}}\left[\int_{0}^{1} t(1-t) f^{\prime \prime}\left(t A_{n, k}+(1-t) A_{n, k+1}\right) d t\right]\right| \\
& \leq \sum_{k=1}^{n} \frac{(b-a)^{2}}{2 n^{3}}\left[\int_{0}^{1}|t(1-t)|\left|f^{\prime \prime}\left(t A_{n, i}+(1-t) A_{n, i+1}\right)\right| d t\right] .
\end{aligned}
$$

Since $\left|f^{\prime \prime}\right|$ is an arithmetic-harmonically convex function on the interval $[a, b]$, the inequality

$$
\left|f^{\prime \prime}\left(t A_{n, k}+(1-t) A_{n, k+1}\right)\right| \leq \frac{\left|f^{\prime \prime}\left(A_{n, k}\right)\right|\left|f^{\prime \prime}\left(A_{n, k+1}\right)\right|}{t\left|f^{\prime \prime}\left(A_{n, k+1}\right)\right|+(1-t)\left|f^{\prime \prime}\left(A_{n, k}\right)\right|}
$$

holds. By using the above inequality in (2.3), the inequality

$$
\left|J_{n}(f, a, b)\right|=\leq \sum_{k=1}^{n} \frac{(b-a)^{2}}{2 n^{3}} \int_{0}^{1} \frac{t(1-t)\left|f^{\prime \prime}\left(A_{n, k}\right)\right|\left|f^{\prime \prime}\left(A_{n, k+1}\right)\right|}{t\left|f^{\prime \prime}\left(A_{n, k+1}\right)\right|+(1-t)\left|f^{\prime \prime}\left(A_{n, k}\right)\right|} d t
$$

is obtained. By changing variable as $u=t\left|f^{\prime \prime}\left(A_{n, k+1}\right)\right|+(1-t)\left|f^{\prime \prime}\left(A_{n, k}\right)\right|$ in the last integral, it is easily seen that

$$
\begin{aligned}
& \int_{0}^{1} \frac{t(1-t)}{t\left|f^{\prime \prime}\left(A_{n, k+1}\right)\right|+(1-t)\left|f^{\prime \prime}\left(A_{n, k}\right)\right|} d t=\frac{1}{\left(\left|f^{\prime \prime}\left(A_{n, k+1}\right)\right|-\left|f^{\prime \prime}\left(A_{n, k}\right)\right|\right)^{3}} \int_{\left|f^{\prime \prime}\left(A_{n, k}\right)\right|}^{\left|f^{\prime \prime}\left(A_{n, k+1}\right)\right|} \frac{\left(u-\left|f^{\prime \prime}\left(A_{n, k}\right)\right|\right)\left(\left|f^{\prime \prime}\left(A_{n, k+1}\right)\right|-u\right)}{u} d u \\
& =\left.\frac{\left[-\frac{u^{2}}{2}+\left(\left|f^{\prime \prime}\left(A_{n, k+1}\right)\right|+\left|f^{\prime \prime}\left(A_{n, k}\right)\right|\right) u-\left|f^{\prime \prime}\left(A_{n, k}\right)\right|\left|f^{\prime \prime}\left(A_{n, k+1}\right)\right| \ln u\right]}{\left(\left|f^{\prime \prime}\left(A_{n, k+1}\right)\right|-\left|f^{\prime \prime}\left(A_{n, k}\right)\right|\right)^{3}}\right|_{\left|f^{\prime \prime}\left(A_{n, k}\right)\right|} ^{\left|f^{\prime \prime}\left(A_{n, k+1}\right)\right|} \\
& =\frac{1}{\left(\left|f^{\prime \prime}\left(A_{n, k+1}\right)\right|-\left|f^{\prime \prime}\left(A_{n, k}\right)\right|\right)^{3}}\left[-\frac{\left|f^{\prime \prime}\left(A_{n, k+1}\right)\right|^{2}-\left|f^{\prime \prime}\left(A_{n, k}\right)\right|^{2}}{2}\right. \\
& +\left(\left|f^{\prime \prime}\left(A_{n, k+1}\right)\right|+\left|f^{\prime \prime}\left(A_{n, k}\right)\right|\right)\left(\left|f^{\prime \prime}\left(A_{n, k+1}\right)\right|-\left|f^{\prime \prime}\left(A_{n, k}\right)\right|\right) \\
& \text { - } \left.\left|f^{\prime \prime}\left(A_{n, k}\right)\right|\left|f^{\prime \prime}\left(A_{n, k+1}\right)\right|\left(\ln \left|f^{\prime \prime}\left(A_{n, k+1}\right)\right|-\ln \left|f^{\prime \prime}\left(A_{n, k}\right)\right|\right)\right] \\
& =\frac{A\left(\left|f^{\prime \prime}\left(A_{n, k}\right)\right|,\left|f^{\prime \prime}\left(A_{n, k+1}\right)\right|\right)-\left|f^{\prime \prime}\left(A_{n, k}\right)\right|\left|f^{\prime \prime}\left(A_{n, k+1}\right)\right| L^{-1}\left(\left|f^{\prime \prime}\left(A_{n, k}\right)\right|,\left|f^{\prime \prime}\left(A_{n, k+1}\right)\right|\right)}{\left(\left|f^{\prime \prime}\left(A_{n, k+1}\right)\right|-\left|f^{\prime \prime}\left(A_{n, k}\right)\right|\right)^{2}}
\end{aligned}
$$

Substituting (2.5) in (2.4), the inequality

$$
\begin{gathered}
\left|J_{n}(f, a, b)\right| \leq \sum_{k=1}^{n} \frac{(b-a)^{2}}{2 n^{3}} \frac{\left|f^{\prime \prime}\left(A_{n, k}\right)\right|\left|f^{\prime \prime}\left(A_{n, k+1}\right)\right|}{\left(\left|f^{\prime \prime}\left(A_{n, k+1}\right)\right|-\left|f^{\prime \prime}\left(A_{n, k}\right)\right|\right)^{2}} \\
\times\left[A\left(\left|f^{\prime \prime}\left(A_{n, k}\right)\right|,\left|f^{\prime \prime}\left(A_{n, k+1}\right)\right|\right)-\left|f^{\prime \prime}\left(A_{n, k}\right)\right|\left|f^{\prime \prime}\left(A_{n, k+1}\right)\right| L^{-1}\left(\left|f^{\prime \prime}\left(A_{n, k}\right)\right|,\left|f^{\prime \prime}\left(A_{n, k+1}\right)\right|\right)\right],
\end{gathered}
$$

is obtained which is the desired result.

ii) Let $\left|f^{\prime \prime}\left(A_{n, k+1}\right)\right|-\left|f^{\prime \prime}\left(A_{n, k}\right)\right|=0$. Then, substituting $\left|f^{\prime \prime}\left(A_{n, k+1}\right)\right|=\left|f^{\prime \prime}\left(A_{n, k}\right)\right|$ in the inequality (2.4), the following holds:

$$
\left|J_{n}(f, a, b)\right| \leq \sum_{k=1}^{n} \frac{(b-a)^{2}}{12 n^{3}}\left|f^{\prime \prime}\left(A_{n, k+1}\right)\right| .
$$

This completes the proof of theorem. 
Corollary 2.2. By choosing $n=1$ in Theorem 2.1, the following inequalities are obtained: i) If $\left|f^{\prime \prime}\left(A_{1, k+1}\right)\right|-\left|f^{\prime \prime}\left(A_{1, k}\right)\right| \neq 0$ for $k=1$, then

$$
\left|\frac{f(a)+f(b)}{2}-\frac{1}{b-a} \int_{a}^{b} f(x) d x\right| \leq \frac{(b-a)^{2}}{2} \frac{\left|f^{\prime \prime}(a)\right|\left|f^{\prime \prime}(b)\right|\left[A\left(\left|f^{\prime \prime}(a)\right|,\left|f^{\prime \prime}(b)\right|\right)-\left|f^{\prime \prime}(a)\right|\left|f^{\prime \prime}(b)\right| L^{-1}\left(\left|f^{\prime \prime}(a)\right|,\left|f^{\prime \prime}(b)\right|\right)\right]}{\left(\left|f^{\prime \prime}(b)\right|-\left|f^{\prime \prime}(a)\right|\right)^{2}}
$$

ii) If $\left|f^{\prime \prime}\left(A_{1, k+1}\right)\right|-\left|f^{\prime \prime}\left(A_{1, k}\right)\right|=0$ for $k=1$, then

$$
\left|\frac{f(a)+f(b)}{2}-\frac{1}{b-a} \int_{a}^{b} f(x) d x\right| \leq \frac{(b-a)^{2}}{12}\left|f^{\prime \prime}(b)\right| .
$$

Corollary 2.3. By choosing $n=2$ in Theorem 2.1, the following Bullen type inequalities are obtained: i) If $\left|f^{\prime \prime}\left(A_{2, k+1}\right)\right|-\left|f^{\prime \prime}\left(A_{2, k}\right)\right| \neq 0$ for $k=1,2$, then

$$
\begin{aligned}
\left|\frac{1}{2}\left[\frac{f(a)+f(b)}{2}+f\left(\frac{a+b}{2}\right)\right]-\frac{1}{b-a} \int_{a}^{b} f(x) d x\right| & \leq \frac{(b-a)^{2}}{16} \frac{\left|f^{\prime \prime}(a)\right|\left|f^{\prime \prime}\left(\frac{a+b}{2}\right)\right|}{\left(\left|f^{\prime \prime}\left(\frac{a+b}{2}\right)\right|-\left|f^{\prime \prime}(a)\right|\right)^{2}}\left[A\left(\left|f^{\prime \prime}(a)\right|,\left|f^{\prime \prime}\left(\frac{a+b}{2}\right)\right|\right)\right. \\
& \left.-\left|f^{\prime \prime}(a)\right|\left|f^{\prime \prime}\left(\frac{a+b}{2}\right)\right|-L^{-1}\left(\left|f^{\prime \prime}(a)\right|,\left|f^{\prime \prime}\left(\frac{a+b}{2}\right)\right|\right)\right] \\
& +\frac{(b-a)^{2}}{16} \frac{\left|f^{\prime \prime}\left(\frac{a+b}{2}\right)\right|\left|f^{\prime \prime}(b)\right|}{\left(\left|f^{\prime \prime}(b)\right|-\left|f^{\prime \prime}\left(\frac{a+b}{2}\right)\right|\right)^{2}}\left[A\left(\left|f^{\prime \prime}\left(\frac{a+b}{2}\right)\right|,\left|f^{\prime \prime}(b)\right|\right)\right. \\
& \left.-\left|f^{\prime \prime}\left(\frac{a+b}{2}\right)\right|\left|f^{\prime \prime}(b)\right|-L^{-1}\left(\left|f^{\prime \prime}\left(\frac{a+b}{2}\right)\right|,\left|f^{\prime \prime}(b)\right|\right)\right],
\end{aligned}
$$

ii) If $\left|f^{\prime \prime}\left(A_{2, k+1}\right)\right|-\left|f^{\prime \prime}\left(A_{2, k}\right)\right|=0$ for $k=1,2$, then

$$
\left|\frac{1}{2}\left[\frac{f(a)+f(b)}{2}+f\left(\frac{a+b}{2}\right)\right]-\frac{1}{b-a} \int_{a}^{b} f(x) d x\right| \leq \frac{(b-a)^{2}}{96}\left[\left|f^{\prime \prime}\left(\frac{a+b}{2}\right)\right|+\left|f^{\prime \prime}(b)\right|\right] .
$$

Theorem 2.4. Let $f: I \subset(0, \infty) \rightarrow(0, \infty)$ be a twice differentiable mapping on $I^{\circ}, n \in \mathbb{N}$ and $a, b \in I^{\circ}$ with $a<b$ such that $f^{\prime \prime} \in L_{1}[a, b]$ and $\left|f^{\prime \prime}\right|^{q}$ are an arithmetic-harmonically convex function on the interval $[a, b]$ for some fixed $q>1$, then the following inequalities hold: i) If $\left|f^{\prime \prime}\left(A_{n, k+1}\right)\right|^{q}-\left|f^{\prime \prime}\left(A_{n, k}\right)\right|^{q} \neq 0$, then

$$
\left|J_{n}(f, a, b)\right| \leq \sum_{k=1}^{n} \frac{(b-a)^{2}}{2 n^{3}} \frac{[B(p+1, p+1)]^{\frac{1}{p}} G^{2}\left(\left|f^{\prime \prime}\left(A_{n, k}\right)\right|,\left|f^{\prime \prime}\left(A_{n, k+1}\right)\right|\right)}{L^{\frac{1}{q}}\left(\left|f^{\prime \prime}\left(A_{n, k}\right)\right|^{q},\left|f^{\prime \prime}\left(A_{n, k+1}\right)\right|^{q}\right)},
$$

ii) If $\left|f^{\prime \prime}\left(A_{n, k+1}\right)\right|^{q}-\left|f^{\prime \prime}\left(A_{n, k}\right)\right|^{q}=0$, then

$$
\left|J_{n}(f, a, b)\right| \leq \sum_{k=1}^{n} \frac{(b-a)^{2}}{2 n^{3}}[B(p+1, p+1)]^{\frac{1}{p}}\left|f^{\prime \prime}\left(A_{n, k+1}\right)\right| .
$$

where $B(\alpha, \beta)$ is the classical Beta function and $\frac{1}{p}+\frac{1}{q}=1$.

Proof. i) Let $\left|f^{\prime \prime}\left(A_{n, k+1}\right)\right|^{q}-\left|f^{\prime \prime}\left(A_{n, k}\right)\right|^{q} \neq 0$. From the Lemma 1.5 and the properties of modulus, the following inequality can be writtten

$$
\left|J_{n}(f, a, b)\right| \leq \sum_{k=1}^{n} \frac{(b-a)^{2}}{2 n^{3}}\left[\int_{0}^{1}|t(1-t)|\left|f^{\prime \prime}\left(t A_{n, k}+(1-t) A_{n, k+1}\right)\right| d t\right] .
$$

Since $\left|f^{\prime \prime}\right|^{q}$ is an arithmetic-harmonically convex function on the interval $[a, b]$, the inequality

$$
\left|f^{\prime \prime}\left(t A_{n, k}+(1-t) A_{n, k+1}\right)\right|^{q} \leq \frac{\left|f^{\prime \prime}\left(A_{n, k}\right)\right|^{q}\left|f^{\prime \prime}\left(A_{n, k+1}\right)\right|^{q}}{t\left|f^{\prime \prime}\left(A_{n, k+1}\right)\right|^{q}+(1-t)\left|f^{\prime \prime}\left(A_{n, k}\right)\right|^{q}}
$$

holds. By applying the well known Hölder integral inequality and the inequality (2.9) on (2.8), the inequality

$$
\begin{aligned}
\left|J_{n}(f, a, b)\right| & \leq \sum_{k=1}^{n} \frac{(b-a)^{2}}{2 n^{3}}\left(\int_{0}^{1}[t(1-t)]^{p} d t\right)^{\frac{1}{p}}\left(\int_{0}^{1}\left|f^{\prime \prime}\left(t A_{n, k}+(1-t) A_{n, k+1}\right)\right|^{q} d t\right)^{\frac{1}{q}} \\
& \leq \sum_{k=1}^{n} \frac{(b-a)^{2}}{2 n^{3}}\left(\int_{0}^{1} t^{p}(1-t)^{p} d t\right)^{\frac{1}{p}}\left(\int_{0}^{1} \frac{\left|f^{\prime \prime}\left(A_{n, k}\right)\right|^{q}\left|f^{\prime \prime}\left(A_{n, k+1}\right)\right|^{q} d t}{t\left|f^{\prime \prime}\left(A_{n, k+1}\right)\right|^{q}+(1-t)\left|f^{\prime \prime}\left(A_{n, k}\right)\right|^{q}}\right)^{\frac{1}{q}} \\
& =\sum_{k=1}^{n} \frac{(b-a)^{2}}{2 n^{3}} \frac{[B(p+1, p+1)]^{\frac{1}{p}} G^{2}\left(\left|f^{\prime \prime}\left(A_{n, k}\right)\right|,\left|f^{\prime \prime}\left(A_{n, k+1}\right)\right|\right)}{L^{\frac{1}{q}}\left(\left|f^{\prime \prime}\left(A_{n, k}\right)\right|^{q},\left|f^{\prime \prime}\left(A_{n, k+1}\right)\right|^{q}\right)}
\end{aligned}
$$


is obtained, where

$$
\begin{aligned}
\int_{0}^{1} t^{p}(1-t)^{p} d t & =B(p+1, p+1) \\
\int_{0}^{1} \frac{1}{t\left|f^{\prime \prime}\left(A_{n, k+1}\right)\right|^{q}+(1-t)\left|f^{\prime \prime}\left(A_{n, k}\right)\right|^{q}} d t & =L^{-1}\left(\left|f^{\prime \prime}\left(A_{n, k}\right)\right|^{q},\left|f^{\prime \prime}\left(A_{n, k+1}\right)\right|^{q}\right) .
\end{aligned}
$$

ii) Let $\left|f^{\prime \prime}\left(A_{n, k+1}\right)\right|^{q}-\left|f^{\prime \prime}\left(A_{n, k}\right)\right|^{q}=0$. Then, substituting $\left|f^{\prime \prime}\left(A_{n, k+1}\right)\right|^{q}=\left|f^{\prime \prime}\left(A_{n, k}\right)\right|^{q}$ in the inequality (2.10), the following inequality is found:

$$
\left|J_{n}(f, a, b)\right| \leq \sum_{k=1}^{n} \frac{(b-a)^{2}}{2 n^{3}}[B(p+1, p+1)]^{\frac{1}{p}}\left|f^{\prime \prime}\left(A_{n, k+1}\right)\right| .
$$

This completes the proof of theorem.

Corollary 2.5. By choosing $n=1$ in Theorem 2.4, the following inequalities are obtained: i) If $\left|f^{\prime \prime}\left(A_{1, k+1}\right)\right|-\left|f^{\prime \prime}\left(A_{1, k}\right)\right| \neq 0$ for $k=1$, then

$$
\left|\frac{f(a)+f(b)}{2}-\frac{1}{b-a} \int_{a}^{b} f(x) d x\right| \leq \frac{(b-a)^{2}}{2} \frac{[B(p+1, p+1)]^{\frac{1}{p}} G^{2}\left(\left|f^{\prime \prime}(a)\right|,\left|f^{\prime \prime}(b)\right|\right)}{L^{\frac{1}{q}}\left(\left|f^{\prime \prime}(a)\right|^{q},\left|f^{\prime \prime}(b)\right|^{q}\right)},
$$

ii) If $\left|f^{\prime \prime}\left(A_{1, k+1}\right)\right|-\left|f^{\prime \prime}\left(A_{1, k}\right)\right|=0$ for $k=1$, then

$$
\left|\frac{f(a)+f(b)}{2}-\frac{1}{b-a} \int_{a}^{b} f(x) d x\right| \leq \frac{(b-a)^{2}}{2}[B(p+1, p+1)]^{\frac{1}{p}}\left|f^{\prime \prime}(b)\right| .
$$

Corollary 2.6. By choosing $n=2$ in Theorem 2.4, the following Bullen type inequalities are obtained: i) If $\left|f^{\prime \prime}\left(A_{2, k+1}\right)\right|-\left|f^{\prime \prime}\left(A_{2, k}\right)\right| \neq 0$ for $k=1,2$, then

$$
\begin{aligned}
\mid \frac{1}{2}\left[\frac{f(a)+f(b)}{2}\right. & \left.+f\left(\frac{a+b}{2}\right)\right]-\frac{1}{b-a} \int_{a}^{b} f(x) d x \mid \leq \frac{(b-a)^{2}}{16}[B(p+1, p+1)]^{\frac{1}{p}} \\
& \times\left[\frac{G^{2}\left(\left|f^{\prime \prime}(a)\right|,\left|f^{\prime \prime}\left(\frac{a+b}{2}\right)\right|\right)}{L^{\frac{1}{q}}\left(\left|f^{\prime \prime}(a)\right|^{q},\left|f^{\prime \prime}\left(\frac{a+b}{2}\right)\right|^{q}\right)}+\frac{G^{2}\left(\left|f^{\prime \prime}\left(\frac{a+b}{2}\right)\right|,\left|f^{\prime \prime}(b)\right|\right)}{L^{\frac{1}{q}}\left(\left|f^{\prime \prime}\left(\frac{a+b}{2}\right)\right|,\left|f^{\prime \prime}(b)\right|\right)}\right],
\end{aligned}
$$

ii) If $\left|f^{\prime \prime}\left(A_{2, k+1}\right)\right|-\left|f^{\prime \prime}\left(A_{2, k}\right)\right|=0$ for $k=1,2$, then

$$
\left|\frac{1}{2}\left[\frac{f(a)+f(b)}{2}+f\left(\frac{a+b}{2}\right)\right]-\frac{1}{b-a} \int_{a}^{b} f(x) d x\right| \leq \frac{(b-a)^{2}}{16}[B(p+1, p+1)]^{\frac{1}{p}}\left[\left|f^{\prime \prime}\left(\frac{a+b}{2}\right)\right|+\left|f^{\prime \prime}(b)\right|\right] .
$$

Theorem 2.7. Let $f: I \subset(0, \infty) \rightarrow(0, \infty)$ be a twice differentiable mapping on $I^{\circ}, n \in \mathbb{N}$ and $a, b \in I^{\circ}$ with $a<b$ such that $f^{\prime \prime} \in L_{1}[a, b]$ and $\left|f^{\prime \prime}\right|^{q}$ are an arithmetic-harmonically convex function on the interval $[a, b]$ for some fixed $q \geq 1$, then the following inequalities hold: i) If $\left|f^{\prime \prime}\left(A_{n, k+1}\right)\right|^{q}-\left|f^{\prime \prime}\left(A_{n, k}\right)\right|^{q} \neq 0$, then

$$
\begin{aligned}
\left|J_{n}(f, a, b)\right| & \leq \sum_{k=1}^{n} \frac{(b-a)^{2}}{2 n^{3}}\left(\frac{1}{6}\right)^{1-\frac{1}{q}} \frac{G^{2}\left(\left|f^{\prime \prime}\left(A_{n, k}\right)\right|,\left|f^{\prime \prime}\left(A_{n, k+1}\right)\right|\right)}{\left(\left|f^{\prime \prime}\left(A_{n, k+1}\right)\right|^{q}-\left|f^{\prime \prime}\left(A_{n, k}\right)\right|^{q}\right)^{\frac{2}{q}}} \\
& \times\left[A\left(\left|f^{\prime \prime}\left(A_{n, k}\right)\right|^{q},\left|f^{\prime \prime}\left(A_{n, k+1}\right)\right|^{q}\right)-\frac{G^{2}\left(\left|f^{\prime \prime}\left(A_{n, k}\right)\right|^{q},\left|f^{\prime \prime}\left(A_{n, k+1}\right)\right|^{q}\right)}{L\left(\left|f^{\prime \prime}\left(A_{n, k}\right)\right|^{q},\left|f^{\prime \prime}\left(A_{n, k+1}\right)\right|^{q}\right)}\right]^{\frac{1}{q}},
\end{aligned}
$$

ii) If $\left|f^{\prime \prime}\left(A_{n, k+1}\right)\right|^{q}-\left|f^{\prime \prime}\left(A_{n, k}\right)\right|^{q}=0$, then

$$
\left|J_{n}(f, a, b)\right| \leq \sum_{k=1}^{n} \frac{(b-a)^{2}}{12 n^{3}}\left|f^{\prime \prime}\left(A_{n, k+1}\right)\right|,
$$

where $\frac{1}{p}+\frac{1}{q}=1$.

Proof. i) Let $\left|f^{\prime \prime}\left(A_{n, k+1}\right)\right|^{q}-\left|f^{\prime \prime}\left(A_{n, k}\right)\right|^{q} \neq 0$. From the Lemma 1.5 and the properties of modulus, the inequality can be written:

$$
\left|J_{n}(f, a, b)\right| \leq \sum_{k=1}^{n} \frac{(b-a)^{2}}{2 n^{3}}\left[\int_{0}^{1}|t(1-t)|\left|f^{\prime \prime}\left(t A_{n, k}+(1-t) A_{n, k+1}\right)\right| d t\right] .
$$

Since $\left|f^{\prime \prime}\right|^{q}$ is an arithmetic-harmonically convex function on the interval $[a, b]$, the inequality

$$
\left|f^{\prime \prime}\left(t A_{n, k}+(1-t) A_{n, k+1}\right)\right|^{q} \leq \frac{\left|f^{\prime \prime}\left(A_{n, k}\right)\right|^{q}\left|f^{\prime \prime}\left(A_{n, k+1}\right)\right|^{q}}{t\left|f^{\prime \prime}\left(A_{n, k+1}\right)\right|^{q}+(1-t)\left|f^{\prime \prime}\left(A_{n, k}\right)\right|^{q}}
$$

holds. By applying the last inequality and the well known power-mean integral inequality on (2.14), the inequality 


$$
\begin{aligned}
\left|J_{n}(f, a, b)\right| & \leq \sum_{k=1}^{n} \frac{(b-a)^{2}}{2 n^{3}}\left(\int_{0}^{1}|t(1-t)| d t\right)^{1-\frac{1}{q}}\left(\int_{0}^{1}|t(1-t)|\left|f^{\prime \prime}\left(t A_{n, k}+(1-t) A_{n, k+1}\right)\right|^{q} d t\right)^{\frac{1}{q}} \\
& \leq \sum_{k=1}^{n} \frac{(b-a)^{2}}{2 n^{3}}\left(\int_{0}^{1} t(1-t) d t\right)^{1-\frac{1}{q}}\left(\int_{0}^{1} \frac{t(1-t)\left|f^{\prime \prime}\left(A_{n, k}\right)\right|^{q}\left|f^{\prime \prime}\left(A_{n, k+1}\right)\right|^{q}}{t\left|f^{\prime \prime}\left(A_{n, k+1}\right)\right|^{q}+(1-t)\left|f^{\prime \prime}\left(A_{n, k}\right)\right|^{q}} d t\right)^{\frac{1}{q}} \\
& =\sum_{k=1}^{n} \frac{(b-a)^{2}}{2 n^{3}}\left|f^{\prime \prime}\left(A_{n, k}\right)\right|\left|f^{\prime \prime}\left(A_{n, k+1}\right)\right|\left(\frac{1}{6}\right)^{1-\frac{1}{q}}\left(\int_{0}^{1} \frac{t(1-t) d t}{t\left|f^{\prime \prime}\left(A_{n, k+1}\right)\right|^{q}+(1-t)\left|f^{\prime \prime}\left(A_{n, k}\right)\right|^{q}}\right)^{\frac{1}{q}} \\
& =\sum_{k=1}^{n} \frac{(b-a)^{2}}{2 n^{3}}\left(\frac{1}{6}\right)^{1-\frac{1}{q}} \frac{G^{2}\left(\left|f^{\prime \prime}\left(A_{n, k}\right)\right|,\left|f^{\prime \prime}\left(A_{n, k+1}\right)\right|\right)}{\left(\left|f^{\prime \prime}\left(A_{n, k+1}\right)\right|^{q}-\left|f^{\prime \prime}\left(A_{n, k}\right)\right|^{q}\right)^{\frac{2}{q}}} \\
& \times\left[A\left(\left|f^{\prime \prime}\left(A_{n, k}\right)\right|^{q},\left|f^{\prime \prime}\left(A_{n, k+1}\right)\right|^{q}\right)-\frac{G^{2}\left(\left|f^{\prime \prime}\left(A_{n, k}\right)\right|^{q},\left|f^{\prime \prime}\left(A_{n, k+1}\right)\right|^{q}\right)}{L\left(\left|f^{\prime \prime}\left(A_{n, k}\right)\right|^{q},\left|f^{\prime \prime}\left(A_{n, k+1}\right)\right|^{q}\right)}\right]^{\frac{1}{q}}
\end{aligned}
$$

is obtained, where

$$
\begin{aligned}
& \int_{0}^{1} t(1-t) d t=\frac{1}{6}, \\
& \int_{0}^{1} \frac{t(1-t)}{t\left|f^{\prime \prime}\left(A_{n, k+1}\right)\right|^{q}+(1-t)\left|f^{\prime \prime}\left(A_{n, k}\right)\right|^{q}} d t=\frac{1}{\left(\left|f^{\prime \prime}\left(A_{n, k+1}\right)\right|^{q}-\left|f^{\prime \prime}\left(A_{n, k}\right)\right|^{q}\right)^{2}} \\
& \times\left[A\left(\left|f^{\prime \prime}\left(A_{n, k}\right)\right|^{q},\left|f^{\prime \prime}\left(A_{n, k+1}\right)\right|^{q}\right)-\frac{G^{2}\left(\left|f^{\prime \prime}\left(A_{n, k}\right)\right|^{q},\left|f^{\prime \prime}\left(A_{n, k+1}\right)\right|^{q}\right)}{L\left(\left|f^{\prime \prime}\left(A_{n, k}\right)\right|^{q},\left|f^{\prime \prime}\left(A_{n, k+1}\right)\right|^{q}\right)}\right]
\end{aligned}
$$

ii) Let $\left|f^{\prime \prime}\left(A_{n, k+1}\right)\right|^{q}-\left|f^{\prime \prime}\left(A_{n, k}\right)\right|^{q}=0$. Then, substituting $\left|f^{\prime \prime}\left(A_{n, k+1}\right)\right|^{q}=\left|f^{\prime \prime}\left(A_{n, k}\right)\right|^{q}$ in the inequality (2.15), the following inequality is found:

$$
\begin{aligned}
\left|J_{n}(f, a, b)\right| & \leq \sum_{k=1}^{n} \frac{(b-a)^{2}}{2 n^{3}}\left(\int_{0}^{1} t(1-t) d t\right)^{1-\frac{1}{q}}\left(\int_{0}^{1} t(1-t)\left|f^{\prime \prime}\left(A_{n, k+1}\right)\right|^{q} d t\right)^{\frac{1}{q}} \\
& =\sum_{k=1}^{n} \frac{(b-a)^{2}}{12 n^{3}}\left|f^{\prime \prime}\left(A_{n, k+1}\right)\right| .
\end{aligned}
$$

This completes the proof of theorem.

Corollary 2.8. By choosing $n=1$ in Theorem 2.7, the following inequalities are obtained: i) If $\left|f^{\prime \prime}\left(A_{1, k+1}\right)\right|^{q}-\left|f^{\prime \prime}\left(A_{1, k}\right)\right|^{q} \neq 0$ for $k=1$, then

$$
\begin{aligned}
\mid \frac{f(a)+f(b)}{2}-\frac{1}{b-a} & \int_{a}^{b} f(x) d x \mid \leq \frac{(b-a)^{2}}{2}\left(\frac{1}{6}\right)^{1-\frac{1}{q}} \frac{G^{2}\left(\left|f^{\prime \prime}(a)\right|,\left|f^{\prime \prime}(b)\right|\right)}{\left(\left|f^{\prime \prime}(b)\right|^{q}-\left|f^{\prime \prime}(a)\right|^{q}\right)^{\frac{2}{q}}} \\
& \times\left[A\left(\left|f^{\prime \prime}(a)\right|^{q},\left|f^{\prime \prime}(b)\right|^{q}\right)-\frac{G^{2}\left(\left|f^{\prime \prime}(a)\right|^{q},\left|f^{\prime \prime}(b)\right|^{q}\right)}{L\left(\left|f^{\prime \prime}(a)\right|^{q},\left|f^{\prime \prime} b\right|^{q}\right)}\right]^{\frac{1}{q}},
\end{aligned}
$$

ii) If $\left|f^{\prime \prime}\left(A_{1, k+1}\right)\right|^{q}-\left|f^{\prime \prime}\left(A_{1, k}\right)\right|^{q}=0$ for $k=1$, then

$$
\left|\frac{f(a)+f(b)}{2}-\frac{1}{b-a} \int_{a}^{b} f(x) d x\right| \leq \frac{(b-a)^{2}}{12}\left|f^{\prime \prime}(b)\right| .
$$

Corollary 2.9. By choosing $n=2$ in Theorem 2.7, the following Bullen type inequalities are obtained: i) If $\left|f^{\prime \prime}\left(A_{2, k+1}\right)\right|^{q}-\left|f^{\prime \prime}\left(A_{2, k}\right)\right|^{q} \neq 0$ for $k=1,2$, then

$$
\begin{aligned}
& \left|\frac{1}{2}\left[\frac{f(a)+f(b)}{2}+f\left(\frac{a+b}{2}\right)\right]-\frac{1}{b-a} \int_{a}^{b} f(x) d x\right| \leq \frac{(b-a)^{2}}{16}\left(\frac{1}{6}\right)^{1-\frac{1}{q}} \frac{G^{2}\left(\left|f^{\prime \prime}(a)\right|,\left|f^{\prime \prime}\left(\frac{a+b}{2}\right)\right|\right)}{\left(\left|f^{\prime \prime}\left(\frac{a+b}{2}\right)\right|^{q}-\left|f^{\prime \prime}(a)\right|^{q}\right)^{\frac{2}{q}}} \\
& \times\left[A\left(\left|f^{\prime \prime}(a)\right|^{q},\left|f^{\prime \prime}\left(\frac{a+b}{2}\right)\right|^{q}\right)-\frac{G^{2}\left(\left|f^{\prime \prime}(a)\right|^{q},\left|f^{\prime \prime}\left(\frac{a+b}{2}\right)\right|^{q}\right)}{L\left(\left|f^{\prime \prime}(a)\right|^{q},\left|f^{\prime \prime}\left(\frac{a+b}{2}\right)\right|^{q}\right)}\right]^{\frac{1}{q}}+\frac{(b-a)^{2}}{16}\left(\frac{1}{6}\right)^{1-\frac{1}{q}} \frac{G^{2}\left(\left|f^{\prime \prime}\left(\frac{a+b}{2}\right)\right|,\left|f^{\prime \prime}(b)\right|\right)}{\left(\left|f^{\prime \prime}(b)\right|^{q}-\left|f^{\prime \prime}\left(\frac{a+b}{2}\right)\right|^{q}\right)^{\frac{2}{q}}} \\
& \times\left[A\left(\left|f^{\prime \prime}\left(\frac{a+b}{2}\right)\right|^{q},\left|f^{\prime \prime}(b)\right|^{q}\right)-\frac{G^{2}\left(\left|f^{\prime \prime}\left(\frac{a+b}{2}\right)\right|^{q},\left|f^{\prime \prime}(b)\right|^{q}\right)}{L\left(\left|f^{\prime \prime}\left(\frac{a+b}{2}\right)\right|^{q},\left|f^{\prime \prime}(b)\right|^{q}\right)}\right]^{\frac{1}{q}},
\end{aligned}
$$

ii) If $\left|f^{\prime \prime}\left(A_{2, k+1}\right)\right|^{q}-\left|f^{\prime \prime}\left(A_{2, k}\right)\right|^{q}=0$ for $k=1,2$, then

$$
\left|\frac{1}{2}\left[\frac{f(a)+f(b)}{2}+f\left(\frac{a+b}{2}\right)\right]-\frac{1}{b-a} \int_{a}^{b} f(x) d x\right| \leq \frac{(b-a)^{2}}{16}\left[\left|f^{\prime \prime}\left(\frac{a+b}{2}\right)\right|+\left|f^{\prime \prime}(b)\right|\right] .
$$


Corollary 2.10. Taking $q=1$ in the inequality (2.12), the following inequality is obtained:

$$
\left|J_{n}(f, a, b)\right| \leq \sum_{k=1}^{n} \frac{(b-a)^{2}}{2 n^{3}} \frac{G^{2}\left(\left|f^{\prime \prime}\left(A_{n, k}\right)\right|,\left|f^{\prime \prime}\left(A_{n, k+1}\right)\right|\right)}{\left(\left|f^{\prime \prime}\left(A_{n, k+1}\right)\right|-\left|f^{\prime \prime}\left(A_{n, k}\right)\right|\right)^{2}} \times\left[A\left(\left|f^{\prime \prime}\left(A_{n, k}\right)\right|,\left|f^{\prime \prime}\left(A_{n, k+1}\right)\right|\right)-\frac{G^{2}\left(\left|f^{\prime \prime}\left(A_{n, k}\right)\right|, f^{\prime \prime}\left(A_{n, k+1}\right)\right)}{L\left(\left|f^{\prime \prime}\left(A_{n, k}\right)\right|,\left|f^{\prime \prime}\left(A_{n, k+1}\right)\right|\right)}\right] .
$$

\section{Applications for special means}

If $p \in(-1,0)$ then the function $f(x)=x^{p}, x>0$ is an arithmetic harmonically-convex [2]. Using this function, the following propositions are obtained:

Proposition 3.1. Let $0<a<b$ and $p \in(-1,0)$. Then, the following inequality holds:

$$
\begin{aligned}
& \frac{1}{(p+1)(p+2)}\left|\sum_{k=1}^{n} \frac{1}{n}\left[\left(A_{n, k}\right)^{p+2}+\left(A_{n, k+1}\right)^{p+2}\right]-L_{p+2}^{p+2}(a, b)\right| \\
& \leq \sum_{k=1}^{n} \frac{(b-a)^{2}}{2 n^{3}} \frac{\left(A_{n, k}\right)^{p}\left(A_{n, k+1}\right)^{p}}{\left[\left(A_{n, k+1}\right)^{p}-\left(A_{n, k}\right)^{p}\right]^{2}}\left[A\left(\left(A_{n, k}\right)^{p},\left(A_{n, k+1}\right)^{p}\right)-\frac{\left(A_{n, k}\right)^{p}\left(A_{n, k+1}\right)^{p}}{L\left(\left(A_{n, k}\right)^{p},\left(A_{n, k+1}\right)^{p}\right)}\right] .
\end{aligned}
$$

Proof. It is known that if $p \in(-1,0)$ then the function $f(x)=\frac{x^{p+2}}{(p+1)(p+2)}, x>0$ is an arithmetic harmonically-convex function. Therefore, the assertion follows from the inequality (2.1) in the Theorem 2.1 , for $f:(0, \infty) \rightarrow \mathbb{R}, f(x)=\frac{x^{p+2}}{(p+1)(p+2)}$.

Corollary 3.2. Taking $n=1$ in Proposition 3.1, the following inequality is obtained:

$$
\begin{aligned}
& \frac{1}{(p+1)(p+2)}\left|A\left(\left(A_{1,1}\right)^{p+2},\left(A_{1,2}\right)^{p+2}\right)-L_{p+2}^{p+2}(a, b)\right| \\
& \leq \frac{(b-a)^{2}}{2} \frac{\left(A_{1,1}\right)^{p}\left(A_{1,2}\right)^{p}}{\left[\left(A_{1,2}\right)^{p}-\left(A_{1,1}\right)^{p}\right]^{2}}\left[A\left(\left(A_{1,1}\right)^{p},\left(A_{1,2}\right)^{p}\right)-\frac{a^{p} b^{p}}{L\left(a^{p}, b^{p}\right)}\right],
\end{aligned}
$$

that is,

$$
\frac{1}{(p+1)(p+2)}\left|A\left(a^{p+1}, b^{p+1}\right)-L_{p+2}^{p+2}(a, b)\right| \leq \frac{(b-a)^{2}}{2} \frac{a^{p} b^{p}}{\left[a^{p}-b^{p}\right]^{2}}\left[A\left(a^{p}, b^{p}\right)-\frac{G^{2 p}(a b)}{L\left(a^{p}, b^{p}\right)}\right] .
$$

Proposition 3.3. Let $a, b \in(0, \infty)$ with $a<b, q>1$ and $m \in(-1,0)$. Then, the following inequality is obtained:

$$
\frac{1}{\left(\frac{m}{q}+1\right)\left(\frac{m}{q}+2\right)}\left|\sum_{k=1}^{n} \frac{1}{n} A\left(\left(A_{n, k}\right)^{\frac{m}{q}+2},\left(A_{n, k+1}\right)^{\frac{m}{q}+2}\right)-L_{\frac{m}{q}+2}^{\frac{m}{q}+2}(a, b)\right| \leq \sum_{k=1}^{n} \frac{(b-a)^{2}}{2 n^{3}} \frac{[B(p+1, p+1)]^{\frac{1}{p}} G^{\frac{2 m}{q}}\left(\left(A_{n, k}\right),\left(A_{n, k+1}\right)\right)}{L^{\frac{1}{q}}\left(\left(A_{n, k}\right)^{m},\left(A_{n, k+1}\right)^{m}\right)} .
$$

Proof. The assertion follows from the inequality (2.6) in the Theorem 2.4. Let

$$
f(x)=\frac{1}{\left(\frac{m}{q}+1\right)\left(\frac{m}{q}+2\right)} x^{\frac{m}{q}+2}, x \in(0, \infty) .
$$

Then

$$
\left|f^{\prime \prime}(x)\right|^{q}=x^{m}
$$

is an arithmetic harmonically-convex on $(0, \infty)$ and the result follows directly from Theorem 2.4.

Corollary 3.4. Taking $n=1$ in Proposition 3.3, the following inequality is obtained:

$$
\frac{1}{\left(\frac{m}{q}+1\right)\left(\frac{m}{q}+2\right)}\left|A\left(a^{\frac{m}{q}+2}, b^{\frac{m}{q}+2}\right)-L_{\frac{m}{q}+2}^{\frac{m}{q}+2}(a, b)\right| \leq \frac{(b-a)^{2}}{2} \frac{[B(p+1, p+1)]^{\frac{1}{p}} G^{\frac{2 m}{q}}(a, b)}{L^{\frac{1}{q}}\left(a^{m}, b^{m}\right)} .
$$

\section{References}

[1] K. Bekar, Inequalities for three-times differentiable arithmetic-harmonically functions, Turkish J. Anal. Number Theory, (Accepted for publication), (2019).

[2] S.S. Dragomir, Inequalities of Hermite-Hadamard type for AH-convex functions, Stud. Univ. Babeş-Bolyai Math. 61(4) (2016), 489-502.

[3] S.S. Dragomir and C.E.M. Pearce, Selected Topics on Hermite-Hadamard Inequalities and Applications, RGMIA Monographs, Victoria University, (2000).

[4] İ. İşcan, T. Toplu and F. Yetgin, Some new inequalities on generalization of Hermite-Hadamard and Bullen type inequalities, Kragujevac J. Math. (Accepted for publication), 2019.

[5] H. Kadakal, Some New Integral Inequalities for n-Times Differentiable Strongly r-Convex Functions, J. Funct. Spaces, Volume 2019, Article ID 1219237, 10 pages, (2019).

[6] H. Kadakal, Hermite-Hadamard type inequalities for two times differentiable arithmetic-harmonically convex functions, Cumhuriyet Sci. J., (Accepted for publication), (2019)

[7] M. Kadakal and İ. İşcan, Some new inequalities for differentiable arithmetic-harmonically convex functions, C. R. Acad. Bulgare Sci, (Submitted to journal), (2019).

[8] S. Maden, H. Kadakal, M. Kadakal and İ. İşcan, Some new integral inequalities for n-times differentiable convex and concave functions. J. Nonlinear Sci. Appl., 10 (2017), 6141-6148.

[9] M. Z. Sarikaya and N. Aktan, On the generalization of some integral inequalities and their applications, Math. Comput. Model. Dyn. Syst., 54, (2011), 2175-2182.

[10] T. Y. Zhang and F. Qi, Integral Inequalities of Hermite-Hadamard Type for m-AH Convex Functions, Turkish J. Anal. Number Theory, 2(3) (2014), 60-64. 